http://garmian.edu.krd

https://doi.org/10.24271/garmian.128

\title{
On Rad - Supplemented Modules, Weak Rad - Supplemented Modules and Completely Weak Rad - Supplemented Modules
}

\author{
Adil Kadir Jabbar ${ }^{1}$ and Bakhtyar Mahmood Rahim ${ }^{2}$ \\ ${ }^{1}$ Department of Mathematics, College of Science, University of Sulaimani, Sulaimani, Iraq \\ ${ }^{2}$ Department of Mathematics, College of Education, University of Sulaimani, Sulaimani, Iraq \\ 1adilkj@gmail.com or adil.jabbar@univsul.edu.iq ²bakhtyar1987@gmail.com
}

\begin{abstract}
In this paper some results that concerning localization of modules are proved. It also, studies the effect of localization on certain types of modules such as Rad-supplemented modules, weak Rad-supplemented modules and completely weak Rad-supplemented modules. Several conditions are given under which certain properties of such types of algebraic structures are preserved under localization.
\end{abstract}

Keywords: Localization of modules, Rad -supplemented modules, weak Rad -supplemented modules, completely weak Rad-supplemented modules and amply Rad -supplemented modules.

\section{Introduction}

Throughout this paper, $R$ is a commutative ring with identity and $M$ is a unitary left $R$-module. By $N \leq M$ we mean $N$ is a submodule of $M$. A submodule $V$ of $M$ is called a small submodule of $M$, denoted by $V \ll M$, if $L \leq M$ is any submodule such that $V+L=M$, then $L=M[12]$, and $V$ is called a supplement (a weak supplement) of $U \leq M$ if $M=U+V$ and $U \cap V \ll V(U \cap V \ll M)$ [8]. Moreover, $M$ is called a supplemented (a weak supplemented) module if every submodule of $M$ has a supplement (a weak supplement) in $M$ [4]. $M$ is called an amply supplemented $R$ - module if for any submodules $U$ and $V$ of $M$ with $M=U+V$ there exists a submodule $K$ of $U$ such that $K \leq V$ [4], and $M$ is called an amply weak supplemented $R$ - module if every submodule of $M$ has amply supplement in $M$ [8] and $V$ is called a $\operatorname{Rad-supplement~or~a~generalized~}$ supplement (a weak Rad -supplement or a generalized weak supplement) of $U$ in $M$ if $M=U+V$ and $U \cap V \leq \operatorname{Rad} V(U \cap V \leq \operatorname{Rad} M)$ [9]. Moreover, $M$ is called a Rad-supplemented or a generalized supplemented (a weakly Rad-supplemented or a generalized weakly supplemented) module if every submodule of $M$ has a Rad-supplement or a generalized supplement (a weak Rad-supplement or a generalized weak supplement) in $M$ [11]. $M$ is called completely weak Rad-supplemented if every submodule of $M$ is weakly Rad-supplemented [9] and it is called amply Rad-supplemented (or generalized amply supplemented) in case $M=U+V$ implies that $U$ has a $\operatorname{Rad}$-supplement 
(or has a generalized supplement) in $V$ [13]. If $r \in R$, then we define $N: r=\{x \in M ; r x \in N\}$ [1]. A submodule $K \leq M$ is called a radical submodule if $\operatorname{Rad}(K)=K . M$ is called a hollow module if every proper submodule of $M$ is small in $M$ [13], and by a hollow radical submodule is meant a submodule which is both hollow and radical. $M$ is called a semi-simple if every submodule of $M$ is a direct summand [5]. For a submodule $K$ of $M$ we define $S_{M}(K)=\{r \in R: r x \in K$, for some $x \notin K$ \} [2]. A non-empty subset $S$ of $R$ is called a multiplicative system in $R$, if $0 \notin S$ and $a, b \in S$ implies $a b \in S$ [6]. If $S$ is a multiplicative system in $R$, then one can obtain an $R_{S}$-module, denoted by $M_{S}$, under the module operations $\frac{x}{s}+\frac{y}{t}=\frac{t x+s y}{s t}$ and $\frac{r}{u} \cdot \frac{x}{s}=\frac{r x}{u s}$, for $\frac{r}{u} \in R_{S}$ and $\frac{x}{s}, \frac{y}{t} \in M_{S}$, so that when we say $M_{S}$ is a module we mean $M_{S}$ is an $R_{S}$-module. In fact, this module $M_{S}$ is known as the localization of $M$ at the multiplicative system $S$ [1].

\section{Some Basic Preliminaries}

The following are some known results on which we depend to prove the main results of this paper.

Corollary 2.1. [3] Let $M$ be an $R$ - module and $P$ a prime ideal of $R$. For submodules $N, L$ of $M$ the following are satisfied.

(1) $(M / N)_{P} \cong M_{P} / N_{P}$.

(2) $(N+L)_{P} \cong N_{P}+L_{P}$.

(3) $(N \cap L)_{P} \cong N_{P} \cap L_{P}$.

Proposition 2.2. [7] Let $L$ and $N$ be submodules of an $R$-module $M$. Then $L \subseteq N$ if and only if $L_{P} \subseteq N_{P}$ for every maximal ideal $P$ of $R$.

Corollary 2.3. [7] Let $L$ and $N$ be submodules of an $R$-module $M$. Then $L=N$ if and only if $L_{P}=N_{P}$, for every maximal ideal $P$ of $R$.

In particular, $N=M$ if and only if $N_{P}=M_{P}$, for every maximal ideal $P$ of $R$.

Lemma 2.4. [11] Let $M$ be an $R$-module and $V$ a Rad -supplement submodule of $U$ in $M$. If $U \cap V$ is a supplement submodule in $U$, then $V$ is supplement submodule in $M$.

\section{Main Results}

First, we give an example of an $R$-module $M$ in which there is a prime ideal $P$ of $R$ and for which $S(K)>\subseteq P$, for all proper submodules $K$ of $M$.

Example. Consider $Z_{8}$ as a $Z$-module, that is take $R=Z$ and $M=Z_{8}$. Clearly $P=\langle 2\rangle$ is a prime ideal of $Z$. The only proper submodules of $Z_{8}$ are $\{0\},\{0,4\},\{0,2,4,6\}$. Now, one can easily calculate $S(\{0\}), S(\{0,4\})$ and $S(\{0,2,4,6\})$ and get that $S(\{0\})=<2>=P \subseteq P$. 
$S(\{0,4\})=<4>\subseteq P$.

$S(\{0,2,4,6\})=<8>\subseteq P$.

Hence, $Z_{8}$ is a $Z$-module, where $P=<2>$ is a prime ideal of $Z$ and such that $S(K) \subseteq P$ for all submodules $K$ of $Z_{8}$.

Now, we prove the first result.

Lemma 3.1. Let $M$ be an $R$-module with submodules $U$ and $V$ of $M$ and $P$ be any maximal ideal of $R$ such that for each proper submodule $K$ of $M$, we have $S(K) \subseteq P$. If $V_{P}$ is a weak Rad-supplement submodule of $U_{P}$ in $M_{P}$, then for a submodule $L^{I}$ of $U_{P}$, there exists a submodule $L$ of $U$ such that $\frac{V+L}{L}$ is a weak Rad-supplement submodule of $\frac{U}{L}$ in $\frac{M}{L}$.

Proof. As $V_{P}$ is a weak $R a d-$ supplement submodule of $U_{P}$ in $M_{P}$, we have $U_{P}+V_{P}=M_{P}$ and $U_{P} \cap V_{P} \leq \operatorname{Rad}\left(M_{P}\right)$, this implies that $(U+V)_{P}=M_{P}$ and $(U \cap V)_{P} \leq \operatorname{Rad}\left(M_{P}\right)$. By using $[1$, Corollary 3.26], we have $(U \cap V)_{P} \leq(\operatorname{Rad} M)_{P}$, so by Corollary 2.3 and Proposition 2.2, we get $U+V=M$ and $U \cap V \leq \operatorname{Rad} M$, so that $V$ is a weak $\operatorname{Rad}$-supplement submodule of $U$ in $M$. Now, since $L^{I} \leq U_{P}$, so by [1, Lemma 3.16], there exists a submodule $L \leq U$ such that $L^{I}=L_{P}$. Thus by [9, Lemma 2.1], we get $\frac{V+L}{L}$ is a weak Rad-supplement submodule of $\frac{U}{L}$ in $\frac{M}{L}$.

In [9, Proposition II.1], it is proved that every factor module of completely weak Rad -supplemented module is also a completely weak Rad-supplemented module. Now, we prove this result by replacing the module with its localization at maximal ideals.

Proposition 3.2. Let $M$ be an $R$-module with a submodule $L$ of $M$ and $P$ be any maximal ideal of $R$ such that for each proper submodule $K$ of $M$ we have $S(K) \subseteq P$. If $M_{P}$ is a completely weak $\operatorname{Rad}$-supplemented $R_{P}-$ module, then $\frac{M}{L}$ is a completely weak $R a d$-supplemented $R$-module.

Proof. Let $\frac{K}{L}$ be a submodule of $\frac{M}{L}$, where $L \leq K \leq M$. Then, $\frac{K_{P}}{L_{P}}$ is a submodule of $\frac{M_{P}}{L_{P}}$, where $L_{P} \leq K_{P} \leq M_{P}$. Let $\frac{U_{P}}{L_{P}}$ be a submodule of $\frac{K_{P}}{L_{P}}$, where $L_{P} \leq U_{P} \leq K_{P}$. Since $M_{P}$ is a completely weak Rad-supplemented $R_{P}$-module, there exists a submodule $V_{P}$ of $K_{P}$ for which $U_{P}+V_{P}=K_{P}$ and $U_{P} \cap V_{P} \leq \operatorname{Rad}\left(K_{P}\right)$. As $V_{P}$ is a weak Rad -supplement of $U_{P}$ in $K_{P}$ and $L_{P} \leq U_{P}$, so by Lemma 3.1, we get that $\frac{V+L}{L}$ is a weak Rad-supplement of $\frac{U}{L}$ in $\frac{K}{L}$. Hence $\frac{K}{L}$ is a weakly Rad-supplemented module. So that, $\frac{M}{L}$ is a completely weak Rad-supplemented $R$-module.

In the next result, we prove that, if the localization of a module at a maximal ideal is completely weak Rad-supplemented, then the module itself is so. 
Proposition 3.3. Let $M$ be an $R$-module and $P$ be any prime ideal of $R$ such that for each proper submodule $K$ of $M$, we have $S(K) \subseteq P$. If $M_{P}$ is a completely weak Rad-supplemented $R_{P}$ - module, then $M$ is also a completely weak Rad-supplemented $R$-module.

Proof. Let $W$ be any submodule of $R$-module $M$ and $K$ be any submodule of $W$. Then, by Proposition 2.2, $W_{P}$ is a submodule of $M_{P}$ and $K_{P}$ is a submodule of $W_{P}$. Since $M_{P}$ is completely weak $R a d-$ supplemented, then, $W_{P}$ is weak Rad-supplemented, therefore, $K_{P}$ has a weak $\operatorname{Rad}$-supplemented in $M_{P}$. This implies that, there exists a submodule $U^{I}$ of $W_{P}$ such that $U^{I}+K_{P}=W_{P}$ and $U^{\prime} \cap K_{P} \leq \operatorname{Rad}\left(W_{P}\right)$. By [1, Lemma 3.16], there exists a submodule $U$ of $W$ such that $U^{\prime}=U_{P}$. That is, $U_{P}+K_{P}=(U+K)_{P}=W_{P} \quad$ and $U_{P} \cap K_{P}=(U \cap K)_{P} \leq \operatorname{Rad}\left(W_{P}\right)$ by Corollary 2.1. By [1, Corollary 3.26] and Proposition 2.3, we obtain $U+K=W$ and $U \cap K \leq \operatorname{Rad}(W)$. Hence $K$ has a weak $\mathrm{Rad}$-supplement in $W$. This implies that, $M$ is a completely weak Rad-supplemented module.

Now, we give the following corollary to the Proposition 3.3.

Corollary 3.4. Let $M$ be an $R$-module and $P$ a maximal ideal of $R$ such that for each proper submodule $K$ of $M$ we have $S(K) \subseteq P$. Let $M=N \oplus L$, where $N, L$ are submodules of $M$. If $M_{P}$ is a completely weak $\operatorname{Rad}$-supplemented $R_{P}$-module, then $N$ and $L$ is also a completely weak Rad-supplemented $R$-module.

Proof. The proof follows directly by Proposition 3.3 and [9, Proposition 2.2].

Now, we give a condition under which, we can extend the result of $[10$, Lemma 3], to the localized modules.

Lemma 3.5. Let $M$ be an $R$-module and $P$ a maximal ideal of $R$ such that for each proper submodule $K$ of $M$, we have $S(K) \subseteq P$. Let $M=U+V$ for submodules $U$ and $V$ of $M$. If $V_{P}$ contains a $R a d-$ supplement submodule of $U_{P}$ in $M_{P}$, then $U \cap V$ has a $R a d$-supplement submodule in $V$.

Proof. Let $K$ be a submodule of $V$, and suppose that a submodule $K_{P}$ of $V_{P}$ is a Rad - supplement of $U_{P}$ in $M_{P}$, then we have $U_{P}+K_{P}=M_{P}$ and $U_{P} \cap K_{P} \leq\left(\operatorname{Rad} K_{P}\right)$, from the modular law, we have $U_{P} \cap V_{P}+K_{P}=V_{P}$, since $K_{P} \leq V_{P}$, then $\left(U_{P} \cap V_{P}\right) \cap K_{P}=U_{P} \cap K_{P} \leq\left(\operatorname{Rad} K_{P}\right)$, by Corollary 2.1, we get $((U \cap V)+K)_{P}=V_{P}$ and $((U \cap V) \cap K)_{P} \leq\left(\operatorname{Rad} K_{P}\right)$ and by [1, Corollary 3.26], we have $\operatorname{Rad}\left(K_{P}\right)=(\operatorname{Rad} K)_{P}$, hence by Corollary 2.3 and Proposition 2.2, we get that $(U \cap V)+K=V$ and $(U \cap V) \cap K \leq \operatorname{Rad} K$. Thus $K$ is a Rad-supplement submodule of $(U \cap V)$ in $V$.

Now, we give a condition under which we can extend the result of [11, Lemma 4], to the localized modules.

Corollary 3.6. Let $M$ be an $R$-module and $V$ a Rad -supplement submodule of $U$ in $M$, let $P$ be any maximal ideal of $R$ such that for each proper submodule $K$ of $M$, 
we have $S(K) \subseteq P$. If $(U \cap V)_{P}$ is a supplement submodule in $U_{P}$, then $V$ is supplement submodule of some submodule in $M$.

Proof. Let $K$ be a submodule of $U$ and $(U \cap V)_{P}$ is a supplement submodule of $K_{P}$ in $U_{P}$. Then we have $K_{P}+(U \cap V)_{P}=U_{P}$ and $K_{P} \cap(U \cap V)_{P} \ll(U \cap V)_{P}$, then by Corollary 2.1, we get $(K+(U \cap V))_{P}=U_{P}$ and $(K \cap(U \cap V))_{P} \ll(U \cap V)_{P}$, hence by Corollary 2.3 and [1, Corollary 3.26], we get $K+(U \cap V)=U$ and $K \cap(U \cap V) \ll(U \cap V)$, it follows that $(U \cap V)$ is supplement submodule of $K$ in $U$. Thus by Lemma 2.4, we get that $V$ is supplement submodule of some submodule of $M$.

In [11, Proposition 6], it is proved that, if every Rad-supplement submodule of a module is Rad-supplemented module, then the module itself is a supplemented module and now we extend this fact to the localized module.

Proposition 3.7. Let $M$ be a reduced module and $P$ a maximal ideal of $R$ such that for each proper submodule $K$ of $M$, we have $S(K) \subseteq P$. If every $\operatorname{Rad}$-supplement submodule of $M_{P}$ is Rad-supplemented, then $M$ is a supplemented module.

Proof. Let $U$ and $V$ be submodules of $M$ and $V_{P}$ a Rad -supplemented submodule of $U_{P}$ in $M_{P}$. Then we have $U_{P}+V_{P}=M_{P}$ and $U_{P} \cap V_{P} \leq \operatorname{Rad} V_{P}$, since $M$ is a reduced $R$ - module, then by [1, Corollary 3.26], we get $M_{P}$ is a reduced $R_{P}$-module, and we have $V_{P}$ is a $R a d$-supplemented, then by [11, Proposition 5], we get $\operatorname{RadV}_{P} \ll V_{P}$, hence $U_{P}+V_{P}=M_{P}$ and $U_{P} \cap V_{P} \leq \operatorname{Rad} V_{P} \ll V_{P}$, then by Corollary 2.1, we get $(U+V)_{P}=M_{P}$ and $(U \cap V)_{P} \ll V_{P}$, hence by Corollary 2.3 and $[1$, Corollary 3.26], we get $U+V=M$ and $U \cap V \ll V$, it follows that $V$ is a supplement submodule of $U$ in $M$. Thus $M$ is supplement $R$-module.

In the following result, we prove that if a module is a sum of two of its submodules for which the localization of one of them is supplemented, then the submodule contains a supplement of the other submodule.

Corollary 3.8. Let $M$ be an $R$-module with submodules $U$ and $V_{\text {of }} M$, and let $P$ be any maximal ideal of $R$ such that for each proper submodule $K$ of $M$, we have $S(K) \subseteq P$, suppose that $M=U+V$. If $V_{P}$ is a supplemented $R_{P}-$ module, then $V$ contains a supplement submodule of $U$ in $M$.

Proof. Let $L$ be a submodule of $V$ and let $L_{P}$ be a supplement of $U_{P} \cap V_{P}$ in $V_{P}$. Then, we have $L_{P}+\left(U_{P} \cap V_{P}\right)=V_{P}$ and $L_{P} \cap\left(U_{P} \cap V_{P}\right) \ll L_{P}$, where $U_{P} \cap L_{P}=L_{P} \cap\left(U_{P} \cap V_{P}\right) \ll L_{P}$, hence by Corollary 2.1, we get $(L+(U+V))_{P}=V_{P}$ and $(U \cap L)_{P}=(L \cap(U \cap V))_{P} \ll V_{P}$ and by Corollary 2.3 and $[1, \quad$ Corollary 3.26], we get that $L+(U \cap V)=V$ and $U \cap L=L \cap(U \cap V) \ll L$, this means that $L$ is a supplement of $(U \cap V)$ in $V$. Now, $M=U+V=U+(U \cap V)+L=U+L$, hence we get $M=U+L$ and $U \cap L \ll L$, it follows that $L$ is a supplement of $U$ in $M$. Thus $V$ contains a supplement of $U$ in $M$. 
In [10, proposition 5], it is proved that if a module is amply Rad-supplemented, then it is a hollow radical module. Now, we give a condition under which, we can extend this result to the localized module.

Proposition 3.9. Let $R$ be a Noetherian ring and $M$ be a simply radical $R$-module. Let $P$ be a maximal ideal of $R$ such that for each proper submodule $K$ of $M$, we have $S(K) \subseteq P$. If $M_{P}$ is an amply $\operatorname{Rad}-$ supplemented $R_{P}$-module, then $M$ is hollow radical $R$-module.

Proof. Let $U$ be a submodule of $M$ and suppose that $U_{P}+V^{I}=M_{P}$ for a submodule $V^{f}$ of $M_{P}$, by [1, Lemma 3.16], there exists a submodule $V \leq M$ such that $V^{I}=V_{P}$, hence we get $U_{P}+V_{P}=M_{P}$. By hypothesis there exists a submodule $L^{I}$ of $V_{P}$ such that $U_{p}+L^{I}=M_{P}$ and $U_{p} \cap L^{I} \leq \operatorname{Rad}\left(L^{I}\right)$, again by [1, Lemma 3.16], there exists a submodule $L \leq V$ such that $L^{\prime}=L_{P}$, hence $U_{P}+L_{P}=M_{P}$ and $U_{P} \cap L_{P} \leq \operatorname{Rad}\left(L_{P}\right) \quad, \quad$ by Corollary 2.1, $\quad(U+L)_{P}=M_{P} \quad$ and $(U \cap L)_{P} \leq \operatorname{Rad}\left(L_{P}\right)$ and by $\left[1\right.$, Corollary 3.26], we have $\operatorname{Rad}\left(L_{P}\right)=(\operatorname{Rad} L)_{P}$, hence by Corollary 2.3 and Proposition 2.2, we get that $M=U+L$ and $U \cap L \leq \operatorname{Rad}(L)$, and since $M$ is simply radical, it follows that $\operatorname{Rad}(L)=L \cap \operatorname{Rad}(M)=L \cap M=L$, so $L$ is a radical submodule, therefore $L=M$ and so $V=M$. Hence, we deduce that $U$ is a small submodule in $M$. Hence, $M$ is a hollow radical $R$-module.

Next, we prove that if every submodule of a localized module is Rad-supplemented, then the module itself is amply Rad-supplemented.

Proposition 3.10. Let $M$ be an $R$-module and $P$ a maximal ideal of $R$ such that for each proper submodule $K$ of $M$, we have $S(K) \subseteq P$. If every submodule of $M_{P}$ is a $\operatorname{Rad}$-supplemented $R_{P}$-module, then $M$ is an amply Rad-supplemented $R$-module.

Proof. Let $N$ be a submodule of $M$ and $L^{I} \leq M_{P}$. Then $N_{P} \leq M_{P}$ and by [1, Lemma 3.16], there exists a submodule $L$ of $M$ such that $L^{I}=L_{P}$, suppose that $M_{P}=N_{P}+L_{P}$, by assumption there exists a submodule $H^{\prime}$ of $L_{P}$ such that $\left(N_{p} \cap L_{P}\right)+H^{I}=L_{P}$ and $\left(N_{P} \cap L_{P}\right) \cap H^{I}=N_{P} \cap H^{I} \leq \operatorname{Rad} H^{I}$, again by [1, Lemma 3.16], there exists a submodule $H \leq L$ such that $H^{I}=H_{P}$, hence $\left(N_{P} \cap L_{P}\right)+H_{P}=L_{P} \quad$ and $\quad\left(N_{P} \cap L_{P}\right) \cap H_{P}=N_{P} \cap H_{P} \leq \operatorname{Rad}\left(H_{P}\right)$. Thus, $L_{P}=H_{P}+\left(N_{P} \cap L_{P}\right) \leq N_{P}+H_{P}$ and hence $M_{P}=N_{P}+L_{P} \leq N_{P}+H_{P}$. Therefore, $M_{P}=N_{P}+H_{P}$ and $N_{P} \cap H_{P} \leq \operatorname{Rad}\left(H_{P}\right)$, by [1, Corollary 3.26], we have $\operatorname{Rad}\left(H_{P}\right)=(\operatorname{Rad} H)_{P}$, hence by Corollary 2.1, we get $M_{P}=(N+H)_{P}$ and $(N \cap H)_{P} \leq(\operatorname{Rad} H)_{P}$ and by Corollary 2.3 and Proposition 2.2, we get $M=N+H$ and $N \cap H=R a d H$. Hence $N$ has a Rad - supplement $H \leq L$. Thus $M$ is an amply Rad-supplemented module.

In [13, Proposition 2.5], it is proved that if a module is a sum of two Rad-supplemented submodules, then the module itself is Rad-supplemented. Now, we extend this result to the localized module. 
Corollary 3.11. Let $N$ and $L$ be $R a d-$ supplemented $R$ - modules and $P$ a maximal ideal of $R$ such that for each proper submodule $K$ of $M$, we have $S(K) \subseteq P$. If $M_{P}=N_{P}+L_{P}$, then $M$ is a $R a d-$ supplemented $R$-module.

Proof. Since, $M_{P}=N_{P}+L_{P}$, then by Corollary 2.1, we have $M_{P}=(N+L)_{P}$ and also by Corollary 2.3, we get $M=N+L$. Hence by [13, Proposition 2.5], we get that $M$ is a Rad-supplemented module.

In [13, Proposition 2.1], it is proved that a Rad-supplemented module which has zero Jacobson radical is semi simple. Now, we prove this result for the localized module.

Corollary 3.12. Let $M$ be a $\operatorname{Rad}$-supplemented $R$-module with a submodule $L$ of $M$ and $P$ a prime ideal of $R$ such that for each proper submodule $K$ of $M$ we have $S(K) \subseteq P$. If $L_{P} \cap \operatorname{Rad} M_{P}=0$, then $L$ is semi simple.

Proof. Since $L_{P} \cap \operatorname{Rad} M_{P}=0$ and by $[1$, Corollary 3.26], we have $\operatorname{Rad}\left(M_{P}\right)=(\operatorname{Rad} M)_{P}$, then by Corollary 2.1, we get $(L \cap \operatorname{Rad} M)_{P}=0$ and by [7, Corollary 2.3], we get $L \cap \operatorname{Rad} M=0$, hence by [13, Proposition 2.1], we get that $L$ is semi simple.

In [13, Proposition 3.2], it is proved that every supplement submodule of a weak Rad-supplemented module is also weak Rad-supplemented. Now, we prove this result for the localized module.

Proposition 3.13. Let $M$ be an $R$-module and $P$ a maximal ideal of $R$ such that for each proper submodule $K$ of $M$, we have $S(K) \subseteq P$. If $M_{P}$ is a weak $\operatorname{Rad}$-supplemented $R$-module, then every supplement submodule of $M$ is weak Rad-supplemented $R$-module.

Proof. Let $K$ be a supplement submodule of $M$. For any submodule $N \leq K$, since $M_{P}$ is a weak Rad - supplemented module, then there exists $L^{I} \leq M_{P}$ such that $M_{P}=N_{P}+L^{I}$ and $N_{P} \cap L^{I} \leq \operatorname{Rad} M_{P}$, by [1, Lemma 3.16], there exists a submodule $L \leq M$ such that $L^{I}=L_{P}$, hence we get $M_{P}=N_{P}+L_{P}$ and $N_{P} \cap L_{P} \leq \operatorname{Rad}\left(M_{P}\right)$ Thus, $K_{P}=K_{P} \cap M_{P}=K_{P} \cap\left(N_{P}+L_{P}\right)=N_{P}+\left(K_{P} \cap L_{P}\right)$ and $N_{P} \cap\left(K_{P} \cap L_{P}\right)=K_{P} \cap\left(N_{P} \cap L_{P}\right) \leq K_{P} \cap \operatorname{Rad}\left(M_{P}\right)=\operatorname{Rad}\left(K_{P}\right)$ by [13, Lemma 1.1]. Hence, $N_{p}+\left(K_{P} \cap L_{P}\right)=K_{P}$ and $N_{p} \cap\left(K_{P} \cap L_{P}\right) \leq \operatorname{Rad}\left(K_{P}\right)$, by [1, Corollary 3.26], we have $\operatorname{Rad}\left(K_{P}\right)=(\operatorname{Rad} K)_{P}$, hence by Corollary 2.1, we get $(N+(K \cap L))_{P}=K_{P}$ and $(N \cap(K \cap L))_{P} \leq(\operatorname{Rad} K)_{P}$, hence by Corollary 2.3, and Proposition 2.2, we get that $N+(K \cap L)=K$ and $N \cap(K \cap L) \leq \operatorname{Rad} K$. Therefore, we get that $K$ is a weak $\operatorname{Rad}$-supplemented $R$-module.

Next, we prove that, if the sum of the localization of two submodules of a module has a Rad-supplement submodule and if one of the submodules is Rad-supplemented, then the other submodule has a Rad-supplement submodule. 
Proposition 3.14. Let $M$ be an $R$-module with submodules $U, V \leq M$, let $U$ be a Rad -supplemented module and $P$ a maximal ideal of $R$ such that for each proper submodule $K$ of $M$ we have $S(K) \subseteq P$. If $U_{P}+V_{P}$ has a Rad-supplement submodule in $M_{P}$, then $V$ has a $\operatorname{Rad}$-supplement submodule in $M$.

Proof. Let $L$ be a submodule of $V$. Since, $U_{P}+V_{P}$ has a $\operatorname{Rad}-$ supplement in $M_{P}$, suppose that $L_{P}$ is a $\mathrm{Rad}-$ supplement of $U_{P}+V_{P}$, hence we get $L_{P}+\left(U_{P}+V_{P}\right)=M_{P}$ and $L_{P} \cap\left(U_{P}+V_{P}\right) \leq \operatorname{Rad}\left(L_{P}\right)$, by [1, Corollary 3.26], we have $\operatorname{Rad}\left(L_{P}\right)=(\operatorname{Rad} L)_{P}$, hence by Corollary 2.1, we get $(L+(U+V))_{P}=M_{P}$ and $(L \cap(U+V))_{P} \leq(\operatorname{Rad} L)_{P}$, and by Corollary 2.3 and Proposition 2.2, we get that $L+(U+V)=M$ and $L \cap(U+V) \leq \operatorname{Rad}(L)$. For $(L+V) \cap U$, since $U$ is a $\operatorname{Rad}$ - supplemented module, there exists $K \leq U$ such that $(L+V) \cap U+K=U$ and $(L+V) \cap K \leq \operatorname{Rad} K$. Thus we have $L+V+K=M$ and $(L+V) \cap K \leq \operatorname{Rad} K$, that is $K$ is a $\operatorname{Rad}-$ supplement of $L+V$ in $M$. It is clear that $(L+K)+V=M$, since $K+V \leq U+V$, $L \cap(K+V) \leq L \cap(U+V) \leq \operatorname{Rad} L \quad, \quad$ we $\quad$ get that $(L+K) \cap V \leq L \cap(K+V)+K \cap(L+V) \leq \operatorname{Rad} L+\operatorname{Rad} K \leq \operatorname{Rad}(L+K)$. Hence we get $(L+K)+V=M$ and $(L+K) \cap V \leq \operatorname{Rad}(L+K)$, that means $L+K$ is a $\operatorname{Rad}$ - supplemented submodule of $V$ in $M$. Thus $V$ has a Rad-supplement submodule in $M$. 


\section{Reference}

[1] Akray, I., Jabbar, A. K. and Sazeedeh, R.: Some Conditions Under Which Certain Types of Modules Possess Localization Property, Journal Duhok university, 15(1), 2012, 144-152.

[2] Atani, S. E. and Darani, A. Y. : Notes on the Primal Submodules, Chiang Mai J. Sci., 35(3), (2008), 399-410.

[3] Atiiyah, M. F. and Macdonald, I. G.: Introduction to Commutative Algebra, Addison-Wesley Publishing Company, 1969.

[4] Bilhan. G.: Amply FWS modules, Journal of Arts and Sciences, Sayi : 4, Aralik 2005.

[5] Clark, J., Lomp, C., Vajana, N. and Wisbauer R.:. Lifting Modules. Birkhauser Verlag Basel, Boston-berlin, 2006.

[6] Jabbar, A. K. and Hassan, N. H. : On Locally Multiplication Modules, International Mathematical Forum, 11(5), 2016, 213-226.

[7] Jabbar, A. K.: A Generalization of Prime and Weakly Prime submodules, Pure Mathematical sciences, 2(1), 2013, 1-11.

[8] Nebiev, C.: Amply weak supplemented modules, International Journal Computational Cogintion, 3(1), 2005, 88-90.

[9] NISANCI, B., TURKMEN, E. and PANCAR, A.: Completely Weak Radsupplemented Modules, International Journal of Computational Cognttion, 7(2), 2009.

[10] Turkmen, E. and Pancar, A.: Characterizations of Rad-supplemented modules, Miskolc Mathematical Notes, 13(2), 2012, 569 - 580.

[11] Turkmen, E. and Pancar, A.: Some properties of Rad-supplemented modules, International Journal of the Physical Sciences, 6(35), 2011, 7904 - 7909.

[12] Turkmen, E. and Pancer, A.: On Radical Supplemented Modules, International Journal of Computational Cognttion, 7(1), 2009.

[13] Wang, Y. and Ding, N.: Generalized Supplemented Modules, Taiwanese Journal of Mathematics, 10(6), 1589-1601, 2006. 